\title{
Radial abundance gradients from planetary nebulae and young objects: Age effects
}

\author{
Walter J. Maciel, Thaise Rodrigues and Roberto Costa \\ Astronomy Department, University of São Paulo \\ Rua do Matão 1226, 05508-090 São Paulo SP, Brazil \\ email: maciel@astro.iag.usp.br - tsrodrigues@usp.br - roberto@astro.iag.usp.br
}

\begin{abstract}
We discuss the effects on the time variation of the gradients resulting from our determinations of the age distributions of CSPN. The main conclusions are: (i) for most CSPN with accurate radial velocities the kinematic ages are usually under $3 \mathrm{Gyr}$, so that the differences between the gradients of these objects and of younger objects are expected to be small; (ii) results based on age-metallicity relations suggest that there is an older PN population for which the gradients are steeper, in agreement with open cluster data.
\end{abstract}

Keywords. planetary nebulae: general, Galaxy: abundances, Galaxy: evolution

\section{Introduction}

Radial abundance gradients in the disks of spiral galaxies can be studied on the basis of young objects (HII regions, OB stars and associations, cepheids) as well as evolved objects (planetary nebulae, open clusters). The magnitude of the gradients is approximately similar in all cases, but there is some controversy regarding the time evolution of the gradients, which is essentially due to uncertainties in the determination of the magnitudes of the average gradients and, especially, of the ages of the objects considered. In order to solve this problem, we have developed several methods to derive approximate ages for the central stars of planetary nebulae (CSPN), so that their gradients can be more accurately attributed to the correct epoch of galactic evolution.

\section{Age distribution of CSPN}

Maciel, Costa \& Idiart (2010) developed several methods based on age-metallicity relations to obtain the age distribution of a sample of CSPN in the galactic disk, most of which are located in the solar neighbourhood. The age distributions were determined with average uncertainties of 1-2 Gyr, and were compared with the expected distribution based both on the observed mass distribution of white dwarfs and on the age distribution derived from available masses of CSPN. It was concluded that most CSPN in the galactic disk have ages under $6 \mathrm{Gyr}$, and that the age distribution is peaked around 2-4 Gyr.

More recently, Maciel, Rodrigues \& Costa (2011) performed an independent determination of the age distribution of the CSPN based on kinematic data. A large sample was considered with accurate radial velocities from Durand et al. (1998). We have also used the correlations between the stellar ages and the velocity dispersion from the GenevaCopenhagen survey, as given by Nordström et al. (2004), Holmberg et al. (2007), and Holmberg et al. (2009). The new distributions confirm the previous results, in the sense that most objects have ages lower than $5 \mathrm{Gyr}$, but suggest generally lower ages for the CSPN compared to the results based on age-metallicity relations. The estimated age uncertainty in this case is about $1 \mathrm{Gyr}$, lower than in the previous determinations. 


\section{Average gradients in the galactic disk}

The magnitudes of the average gradients are usually in the range -0.03 to -0.06 $\mathrm{dex} / \mathrm{kpc}$ for the Milky Way. The uncertainties are about $0.003 \mathrm{dex} / \mathrm{kpc}$, but this is probably a formal value. As a result, there are some controversies in the interpretation of the data, especially concerning the time variation of the gradients. Our recent results from PN and cepheids suggest that their gradients are similar, considering PN with central stars younger than 3 Gyr from Maciel, Rodrigues \& Costa (2011) and cepheids from the sample by Andrievsky and collaborators as given by Luck et al. (2011). The PN gradients are slightly larger than the gradients derived from the younger objects, but the difference seems now marginal compared to our previous investigations, which may explain the similar gradients measured in PN and HII regions in some Local Group galaxies by Stanghellini et al. (2010) and Magrini et al. (2009).

\section{Effects on the time variation of the gradients}

Our previous results on the time variation of the abundance gradients suggested that the PN gradients were generally steeper than the gradients defined by the younger objects. The present results considers the following data: (i) H II regions as in Maciel \& Costa (2009); (ii) OB stars from Daflon \& Cunha (2004); (iii) Cepheids from Luck et al. (2011); (iv) Open clusters from Andreuzzi et al. (2011); (v) Planetary Nebulae, divided into two groups: a younger group, with ages under $3 \mathrm{Gyr}$, from our kinematic data, and an older group, with ages closer to $6 \mathrm{Gyr}$, based on age-metallicity relations. Two main conclusions can be drawn: (1) Considering the younger objects, with ages under 3 Gyr, for which the ages are better determined, it can be concluded that the gradients have not changed appreciably from all objects considered, that is, the radial $[\mathrm{Fe} / \mathrm{H}]$ gradient has been essentially constant during the last 3-4 Gyr, within the uncertainties. These results are supported by the kinematic age distributions for CSPN based on accurate radial velocities. (2) For the older objects, the uncertainties are much higher, especially in view of the difficulty to derive accurate ages for objects older than 5 Gyr. At face value, it appears that there is a steeper increase of the gradients up to about $4 \mathrm{Gyr}$ ago, followed by approximately constant gradients. These results are supported by the age distribution of CSPN based on age-metallicity relations and, especially, by open cluster data. Of course, it is comparatively more difficult to extend this conclusion to older epochs in the galactic evolution, since the age determinations for these objects are probably uncertain. (FAPESP/CNPq)

\section{References}

Andreuzzi, G., Bragaglia, A., Tosi, M., \& Marconi, G., 2011, MNRAS, 412, 1265

Daflon, S. \& Cunha, K., 2004, ApJ, 617, 1115

Durand, S., Acker, A., \& Zijlstra, A., 1998, A\& AS, 132, 13

Holmberg, J., Nordström, B., \& Andersen, J., 2007, A\&A, 475, 519

Holmberg, J., Nordström, B., \& Andersen, J., 2009, A $\& A, 501,941$

Luck, R. E., Andrievsky, S. M., Kovtyukh, V. V., et al., 2011, AJ, 142, 51

Maciel, W. J. \& Costa, R. D. D., 2009, IAU Symp. 254, Electronic publication, CUP

Maciel, W. J., Costa, R. D. D., \& Idiart, T. E. P., 2010, A\&SA, 512, A19

Maciel, W. J., Rodrigues, T. S., \& Costa, R. D. D., 2011, Rev. Mexicana A\&4A, 47, 401

Magrini, L., Stanghellini, L., \& Villaver, E., 2009, ApJ, 696, 729

Nordström, B., Mayor, M., Andersen, J. et al., 2004, A\& A, 418, 989

Stanghellini, L., Magrini, L., Villaver, E., \& Galli, D., 2010, A\& $A, 521$, A3 